\title{
A comparison between PCA and some enhancement filters for denoising
}

\section{astronomical images}

\author{
Loay K. Abood ${ }^{1}$, Raaid N. Hassan ${ }^{2}$ \\ ${ }^{1}$ Department of Computer Science, College of Science, Baghdad University \\ ${ }^{2}$ Department of Astronomy, College of Science, Baghdad University \\ E-mail: raadnoffi742000@yahoo.com
}

\begin{abstract}
This paper includes a comparison between denoising techniques by using statistical approach, principal component analysis with local pixel grouping (PCA-LPG), this procedure is iterated second time to further improve the denoising performance, and other enhancement filters were used. Like adaptive Wiener low pass-filter to a grayscale image that has been degraded by constant power additive noise, based on statistics estimated from a local neighborhood of each pixel. Performs Median filter of the input noisy image, each output pixel contains the Median value in the Mby-N neighborhood around the corresponding pixel in the input image, Gaussian low pass-filter and Order-statistic filter also be used. Experimental results shows LPG-PCA method gives better performance, especially in image fine structure preservation, compared with other general denoising algorithms.
\end{abstract}

Key words

principal component analysis,

local pixel grouping,

Wiener filter,

Median filter,

Gaussian filter,

Order-statistic filter,

Structural Similarity.

Article info.

Received: Sep. 2013

Accepted: Nov. 2013

Published: Dec. 2013

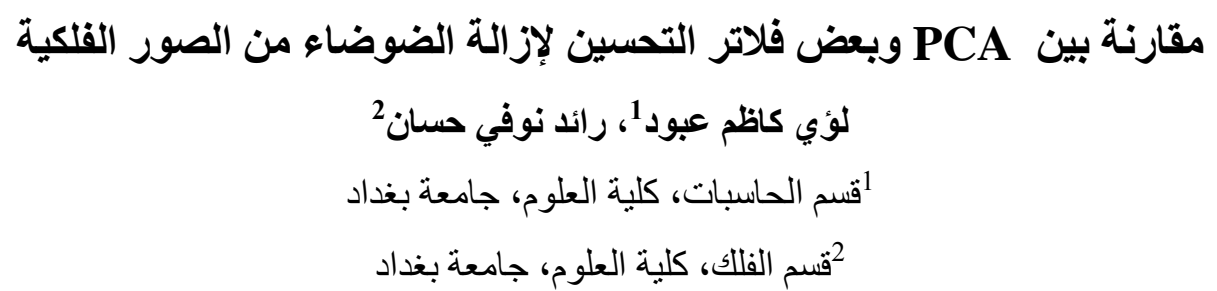

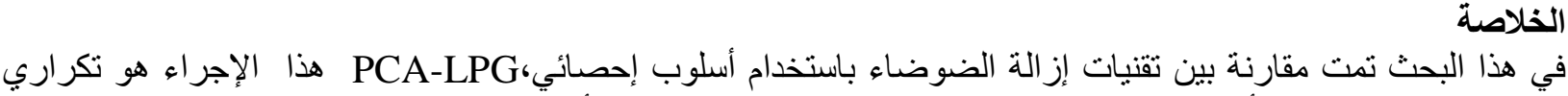

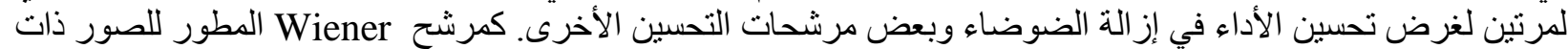

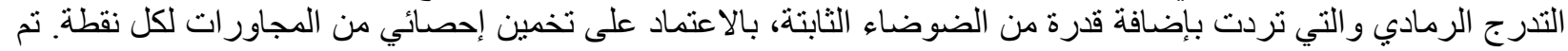

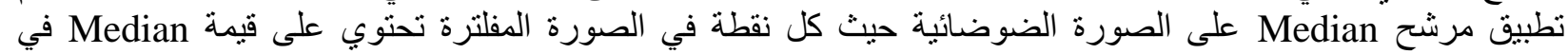

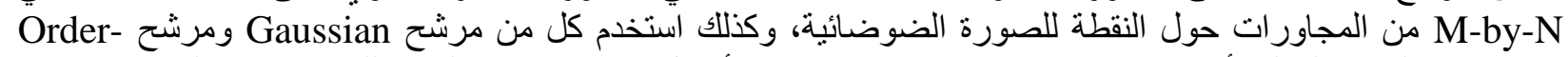

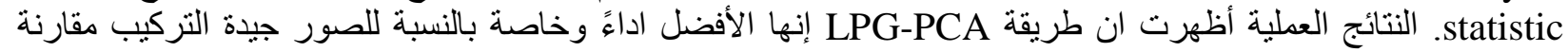
بطرق ازالة الضوضاء الاخرى.

\section{Introduction}

Principal component analysis (PCA) is an orthogonal transformation that seeks the directions of maximum variance in the data and is commonly used to reduce the dimensionality of the data [1].

In image denoising, a compromise has to be found between noise reduction and preserving significant image details. PCA is a statistical technique for simplifying a dataset by reducing datasets to lower dimensions. It is a standard technique commonly used for data reduction in statistical pattern recognition and signal processing [2].

This paper made a comparison between denoising techniques by using statistical approach, principal component analysis with local pixel grouping (PCALPG), this procedure is iterated second time to further improve the denoising performance, and the noise level is adaptively adjusted in the second stage, and other filters were used. Like adaptive 
Wiener low pass-filter to a grayscale image that has been degraded by constant power additive noise, based on statistics estimated from a local neighborhood of each pixel. Performs Median filter of the input noisy image, each output pixel contains the Median value in the M-by-N neighborhood around the corresponding pixel in the input image, Gaussian lowpass-filter and Orderstatistic filter also be used.

\section{Principle components analysis (PCA)}

Real word data sets typically show relationships between variables of their own. These relationships are often linear, or at least nearly so, making it viable for a joint analysis techniques. One of these techniques is the PCA, which rotates the original data to the new coordinates, which makes the data as "flat" as possible.

Due to a table of two or more variables, PCA creates a new table with the same number of variable, called the principal components. Each principle component is a linear transformation of the entire original data set. The coefficients of the principal components are calculated so that the first principal component contains the maximum variance [3, 4].

PCA is fully reversible (original data can be completely restored from the principal components), making it flexible and useful tool to reduce data, noise rejection, visualization and data compression, among other things [5].

\section{Performing Principal Components Analysis \\ Performing PCA can be summarized as follows $[3,6,7]$ :}

1. Determine the size of the data.

2. Calculate the sample mean vector and the sample standard deviation vector to summarize the data.

3. Standardize the data (standardization) by subtracting the sample mean from each observation, then dividing by the sample standard deviation. This centers and scales the data.

4. Determining the coefficients of principal components and their respective variance this done through finding of Eigen vector/value of the covariance matrix.

5. Multiply the standardized data by the principal component coefficients to find the principal components.

6. Now the important thing that is finding the reverse of transformation simply multiplies by the transpose of the coefficient matrix.

7. Go back to the original data, multiply each observation by the sample standard deviation vector and add the mean vector.

This completes the round trip from the original data to the principal components and back to the original data. In some applications, the principal components are modified before the return trip. Interestingly one can note that the first principal component contains nearly $94 \%$ of the variance of the original data [3].

\section{Mathematical representation of the PCA}

Let $Y=\left[y_{1} y_{2} y_{3} \ldots . . y_{m}\right]^{T}$ an m-component vector variable and denoted by $[8,9]$ :

$$
Y=\left[\begin{array}{lll}
y_{11} & y_{12} & y_{13} \ldots \ldots y_{1 n} \\
y_{21} & y_{22} & y_{23} \ldots . . y_{2 n} \\
\vdots & & \\
y_{m 1} & y_{m 2} & y_{m 3} \ldots . . y_{m n}
\end{array}\right]
$$

The sample matrix of $\mathrm{y}$, where $y_{i}^{j}$, $\mathrm{j}=1,2, \ldots \mathrm{n}$, are the discrete samples of variable $y_{i}, i=1,2, \ldots, m$. the $i^{\text {th }}$ row of sample matrix $\mathrm{Y}$, denoted by:

$$
Y_{i}=\left[\begin{array}{llll}
y_{i}^{1} & y_{i}^{2} & \ldots . . & y_{i}^{n}
\end{array}\right]
$$

is called the sample vector of $y_{i}$. The mean value of $Y_{i}$ is calculated as:

$\mu=\frac{1}{n} \sum_{j=1}^{n} Y_{i}(j)$

and the sample vector $Y_{i}$ is centralized matrix of $Y$ is:

$\bar{Y}_{i}=Y_{i}-\mu_{i}=\left[\begin{array}{llll}\bar{y}_{i}^{1} \bar{y}_{i}^{2} & \bar{y}_{i}^{3} & \ldots . . \bar{y}_{i}^{n}\end{array}\right]$

where $\bar{y}_{i}^{j}=y_{i}^{j}-\mu_{i}$. Accordingly, the centralized matrix of $\mathrm{Y}$ is:

$\bar{Y}=\left[\begin{array}{lll}\bar{Y}_{1}^{T} & \bar{Y}_{2}^{T} \bar{Y}_{3}^{T} \ldots \ldots . \bar{Y}_{m}^{T}\end{array}\right]^{T}$

Finally the co-variance matrix of the centralized dataset is calculated as: 


$$
\Omega=\frac{1}{N} \bar{Y} \bar{Y}^{T}
$$

The goal of PCA is to find an orthonormal transformation matrix $\mathrm{P}$ to de-correlate $\bar{Y}$, i.e. $\bar{Z}=P \bar{Y}$ so that the co-variance matrix of the $\mathrm{Z}$ is diagonal. Since the co-variance matrix $\Omega$ is symmetrical, it can be written as:

$\Omega=\phi \Lambda \phi^{T}$

where $\phi=\left[\begin{array}{lllll}\phi_{1} & \phi_{2} & \phi_{3} & \ldots . . . \phi_{m}\end{array}\right]$ is the $\mathrm{m} \times \mathrm{m}$ orthonormal eigenvector matrix and

$\Lambda=\operatorname{diag}\left\{\lambda_{1}, \lambda_{2}, \lambda_{3} \ldots . . \lambda_{m}\right\}$ is the diagonal eigenvalue matrix with $\lambda_{1} \geq \lambda_{2} \geq \lambda_{3} \geq \ldots \ldots . . \geq \lambda_{m}$. By setting:

$P=\phi^{T}$

$\bar{Y}$ can be de-correlated, i.e. $\bar{Z}=P \bar{Y}$ and $\Lambda=\frac{1}{N} \bar{Y} \bar{Y}^{T}$.

In PCA, the energy of a signal will concentrate on a small subset of the PCA transformed dataset, while the energy of noise will evenly spread over the whole dataset. Therefore, the signal and noise can be better distinguished in the PCA domain.

\section{Local Pixel Grouping LPG-PCA denoising algorithm}

An image pixel is described by two quantities, the spatial location and its intensity, while the image local structure is represented as a set of neighboring pixels at different intensity levels. The edge structures convey its, edge preservation semantic information of an image which is highly desired in image denoising [10]. Can be modeled a pixel and its nearest neighbors as a vector variable and perform noise reduction on the vector instead of the single pixel.

PCA was developed by famous personalities the Pearson and the Hotelling, whilst the best modern reference is Jolliffe [11]. Statistically, PCA is a de-correlation technique and it is mainly used in pattern recognition and dimensionality reduction. By transforming the original dataset into PCA domain and preserving only the several most significant principal components, the noise and trivial information can be removed. A PCA-based scheme was proposed for image denoising by using a moving window to calculate the local statistics, from which the local PCA transformation matrix was estimated. As shown in Fig.1, the proposed algorithm has two stages, in the first stage it gives an initial estimation of the image by removing most of the noise and the second stage will further refine the output of the first stage [12]. The second stage has the same type of procedure except for the parameter of noise level. Since the noise in the first stage is significantly reduced, the local pixel grouping (LPG) accuracy will be much improved in the second stage so that the final denoising result is visually much better.

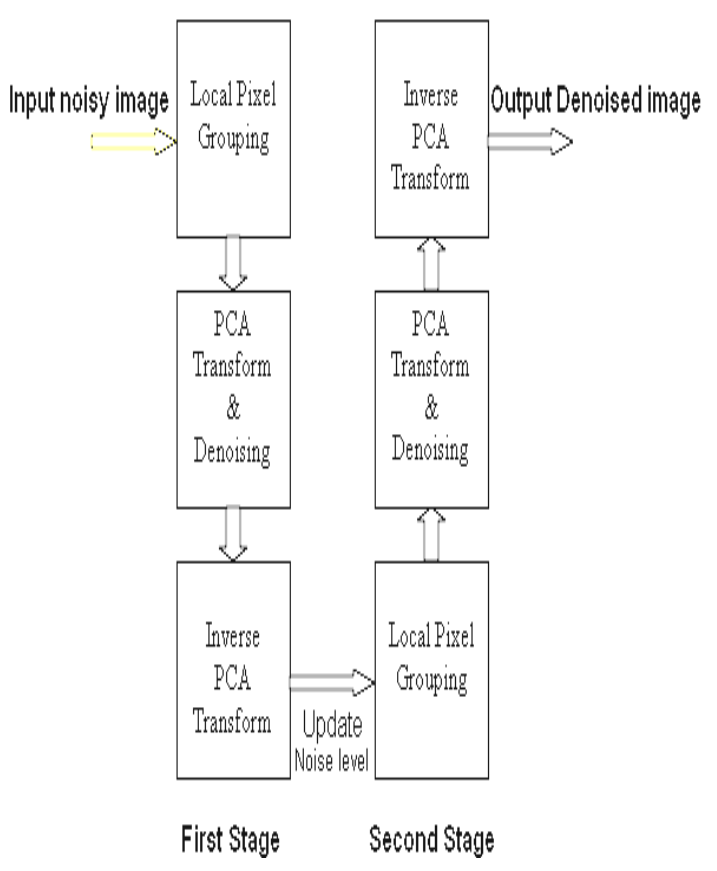

Fig.1: Two stage Principal component analysis

In order to remove the noise from noisy image $Y_{v}$ by using PCA, we need a set of training samples of $Y_{v}$ so that the covariance matrix of $Y_{v}$ and hence the PCA transformation matrix can be calculated .For this purpose, can be used an ( $\mathrm{L} \times \mathrm{L}$ ) training block centered on $Y_{v}$ to find the training samples, as shown in Fig.2. The simplest way is to take the pixels in each possible $(\mathrm{K} \times \mathrm{K})$ block $(\mathrm{K}<\mathrm{L})$ within the $(\mathrm{L}$ $\mathrm{x}$ L) training block as the samples of noisy 
variable $Y_{v}$. In this way, there are totally (L$\mathrm{K}+1)^{2}$ training samples for each component $y_{k}^{v}$ of $Y_{v}$. However, there can be very different blocks from the given central ( $\mathrm{K} \mathrm{X}$ $\mathrm{K})$ block in the ( $\mathrm{L} \times \mathrm{L})$ training window so that taking all the $\mathrm{K} \times \mathrm{K}$ blocks as the training samples of $Y_{v}$ will lead to inaccurate estimation of the covariance matrix of $Y_{v}$, which subsequently leads to inaccurate estimation of the PCA transformation matrix and finally results in much noise residual. Therefore, selecting and grouping the training samples that similar to the central $(\mathrm{K} \times \mathrm{K})$ block is necessary before applying the PCA transform for denoising [2].

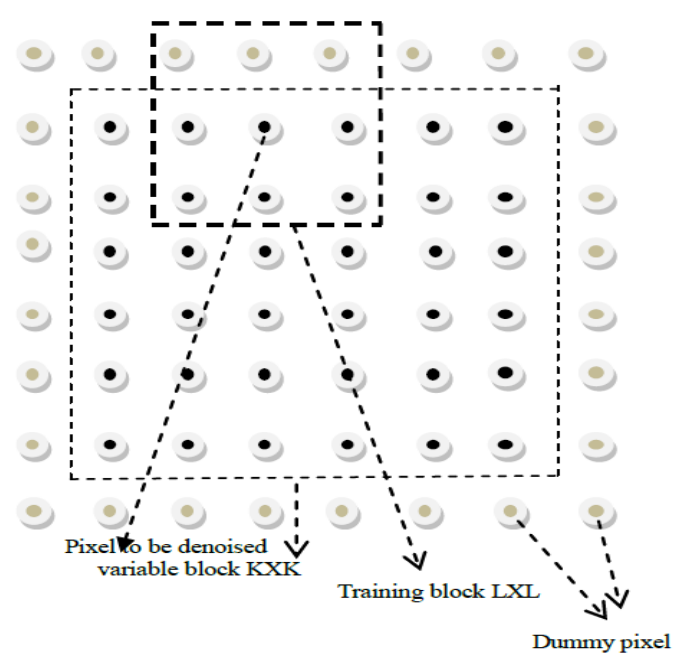

Fig.2: Illustration of the modeling of LPG-PCA based denoising [2].

Wiener adaptive filter: This is a statistical approach to pursue an outcome that can minimize the mean square error between the restored and original image. This approach often produces better results than linear filtering. This adaptive filter is more selective than a comparable linear filter, preserving edges and other high frequency parts of an image. Wiener filters are a class of optimum linear filters which involve linear estimation of a desired signal sequence from another related sequence. The goal of the Wiener adaptive filter is to filter out noise that has corrupted a signal[13].

Gaussian filter: A Gaussian filter is a filter whose impulse response is a Gaussian
Function. Gaussian filters are designed to give no overshoot to a step function input while minimizing the rise and fall time. This behavior is closely connected to the fact that the Gaussian filter has the minimum possible group delay. Mathematically, a Gaussian filter alters the input signal by convolving with a Gaussian function [14].

Median Filter: A median filter belongs to the class of nonlinear filters unlike the mean filter. The median filter follows the moving window principle like the mean filter. A $3 \times 3,5 \times 5$, or $7 \times 7$ kernel of pixels is scanned over pixel matrix of the entire image. The median of the surrounding pixel values in the window is calculated, and the center pixel of the resultant is computed and replaced with the computed median. Median filtering is done by, first sorting all the pixel values from the surrounding neighborhood into numerical order and then replacing the pixel being considered with the middle pixel value [13].

Order statistical filter: Is based on a specific type of image statistics called order statistics. Typically, these filters operate on small subimages, window, and replace the center pixel value (similar to the convolution process). Order statistics a technique that arranges all the pixels in sequential order, based on gray-level value. The placement of the value within this ordered set is referred as the rank [10].

\section{Experimental results and discussions} Evaluate and compared the different denoising techniques (principle component analysis, Weiner filter, Gaussian filter, Median filter and Order statistic filter), using two measures Peak Signal to Noise Ratio (PSNR) and Structural SIMilarity (SSIM) (Under the assumption that human visual perception is highly adapted for extracting structural information from a scene, they introduce an alternative complementary framework for quality assessment based on the degradation of structural information [15]). 
Table 1: The result of two stages LPG- PCA method. PSNR and SSIM values have taken into consideration to compare.

\begin{tabular}{|c|c|c|c|c|}
\hline \multicolumn{5}{|c|}{$\begin{array}{c}\text { Method: LPG-PCA } \\
\text { Image: Point Source (Binary Star) }\end{array}$} \\
\hline \multicolumn{3}{|c|}{ First stage } & \multicolumn{2}{|c|}{ Second Stage } \\
\hline Variance & PSNR_1 & SSIM_1 & PSNR_2 & SSIM_2 \\
\hline 5 & 50.6 & 0.98 & 56.6 & 0.993 \\
\hline 10 & 41.6 & 0.887 & 50.9 & 0.971 \\
\hline 20 & 34.7 & 0.636 & 45.4 & 0.9 \\
\hline 30 & 31 & 0.433 & 41.4 & 0.819 \\
\hline 35 & 29.7 & 0.358 & 39.8 & 0.778 \\
\hline 40 & 28.5 & 0.298 & 38.3 & 0.74 \\
\hline \multicolumn{5}{|c|}{ Image: Satellite } \\
\hline 5 & 39.8 & 0.984 & 39.8 & 0.984 \\
\hline 10 & 34.8 & 0.947 & 34.9 & 0.964 \\
\hline 20 & 29.9 & 0.83 & 29.8 & 0.889 \\
\hline 30 & 27.1 & 0.717 & 27.5 & 0.853 \\
\hline 35 & 26.1 & 0.668 & 26.7 & 0.841 \\
\hline 40 & 25.2 & 0.624 & 26 & 0.829 \\
\hline \multicolumn{5}{|c|}{ Image: Saturn } \\
\hline 5 & 42.3 & 0.979 & 42.8 & 0.983 \\
\hline 10 & 37 & 0.93 & 37.7 & 0.962 \\
\hline 20 & 31.6 & 0.786 & 32.8 & 0.911 \\
\hline 30 & 28.6 & 0.653 & 30.7 & 0.876 \\
\hline 35 & 27.5 & 0.596 & 29.9 & 0.863 \\
\hline 40 & 26.5 & 0.546 & 29.2 & 0.851 \\
\hline
\end{tabular}

LPG-PCA consists of two stages: image estimation by removing the noise and further refinement of the first stage. The noise is significantly reduced in the first stage; the LPG accuracy will be much improved in the second stage so that the final denoising result is visually much better as shown in Table 1. Figs.(3-8) show more explanation by drawing PSNR and SSIM as function of the variance.

Table 2: The comparison of different denoising techniques for different test images.

\begin{tabular}{|c|c|c|c|c|c|c|}
\hline \multirow{2}{*}{ Filter } & \multicolumn{2}{|c|}{ Binary Star } & \multicolumn{2}{c|}{ Satellite } & \multicolumn{2}{c|}{ Saturn } \\
\cline { 2 - 7 } & PSNR & SSIM & PSNR & SSIM & PSNR & SSIM \\
\hline LPG-PCA & 45.4 & 0.993 & 29.8 & 0.889 & 32.8 & 0.911 \\
\hline Wiener & 29.7 & 0.341 & 28 & 0.780 & 29.8 & 0.714 \\
\hline Median & 29.6 & 0.315 & 26.1 & 0.717 & 28.7 & 0.636 \\
\hline Gaussian & 26 & 0.180 & 25.6 & 0.604 & 25.9 & 0.493 \\
\hline Order statistic & 20.1 & 0.02 & 17.3 & 0.505 & 19.25 & 0.278 \\
\hline
\end{tabular}




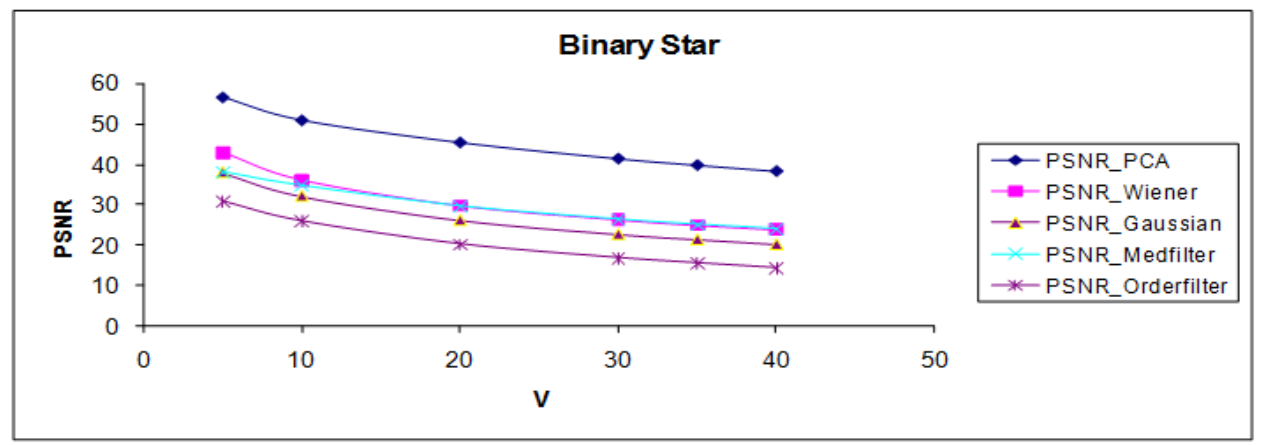

Fig.3: PSNR as a function of variance for binary star.

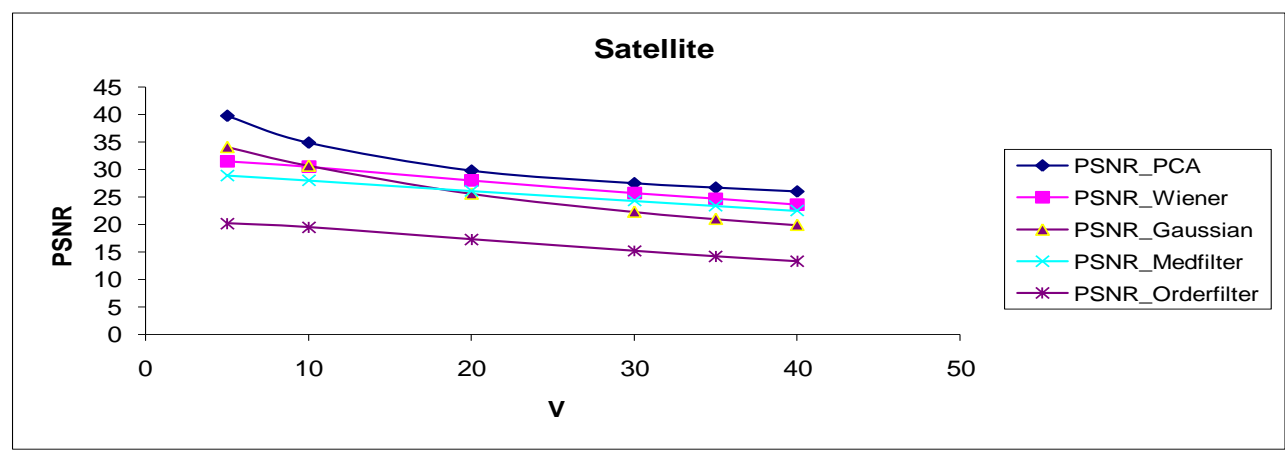

Fig.4: PSNR as a function of variance for Satellite.

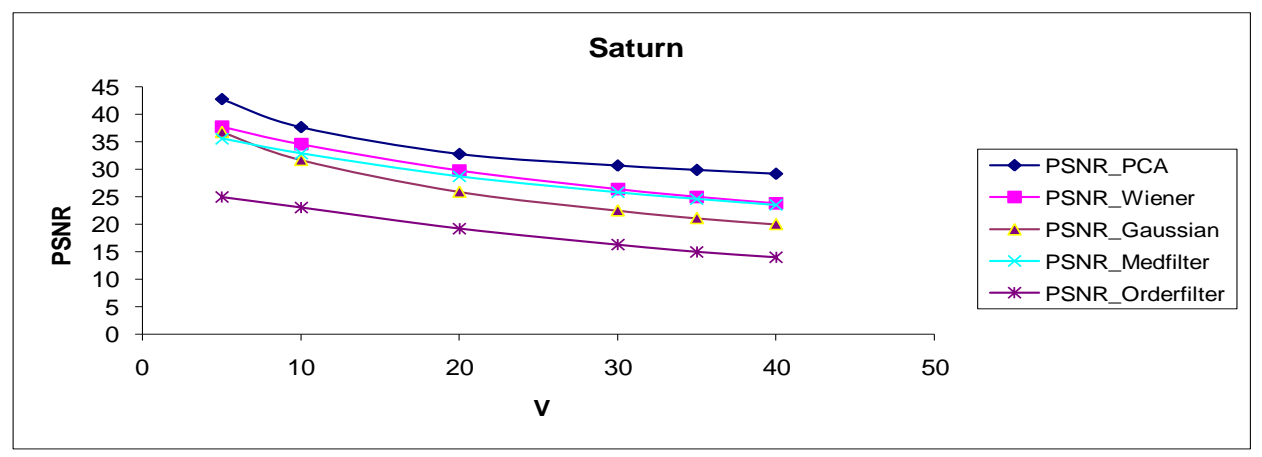

Fig.5: PSNR as a function of variance for Saturn.

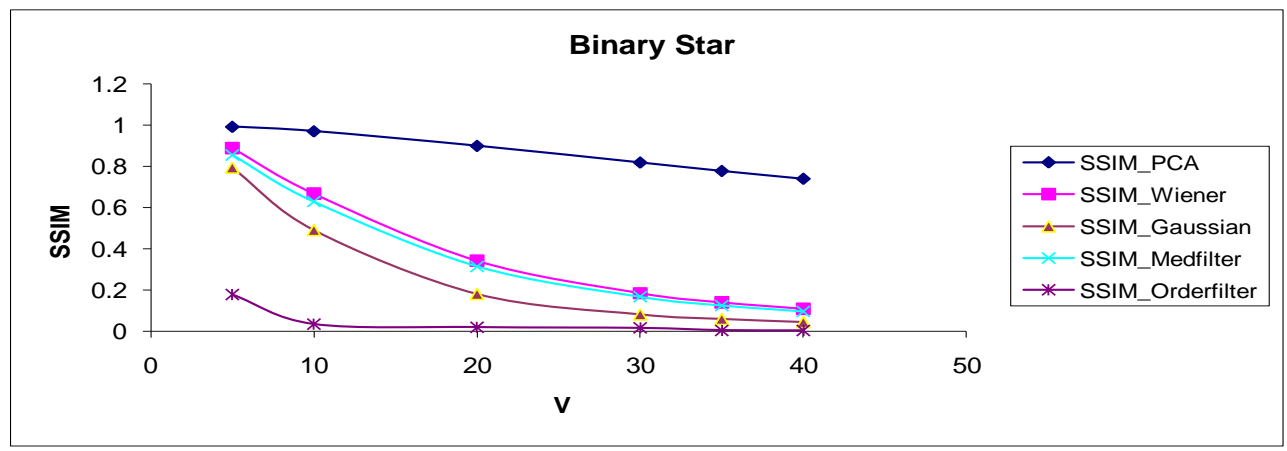

Fig.6: SSIM as a function of variance for binary star. 


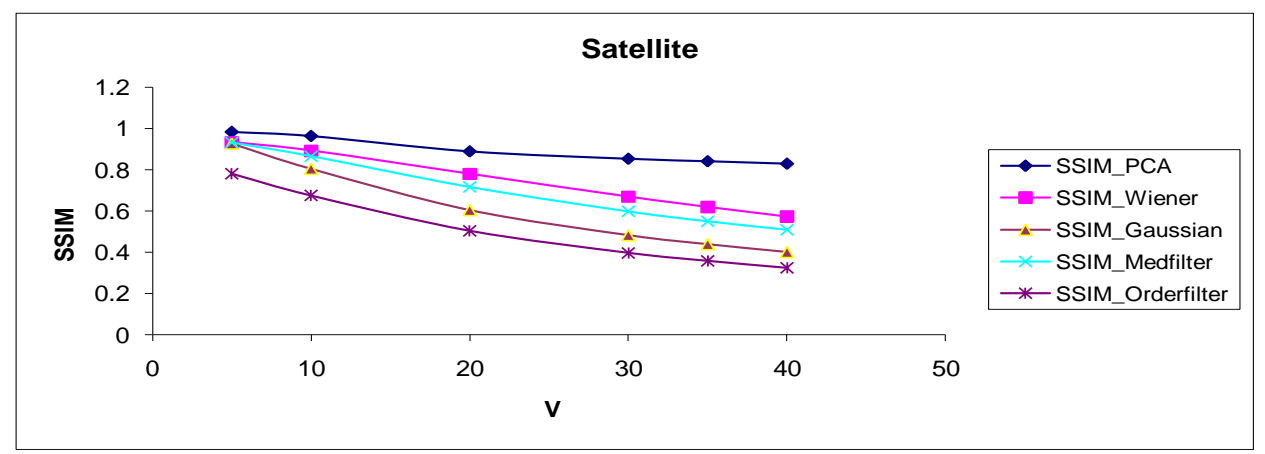

Fig.7: SSIM as a function of variance for Satellite.

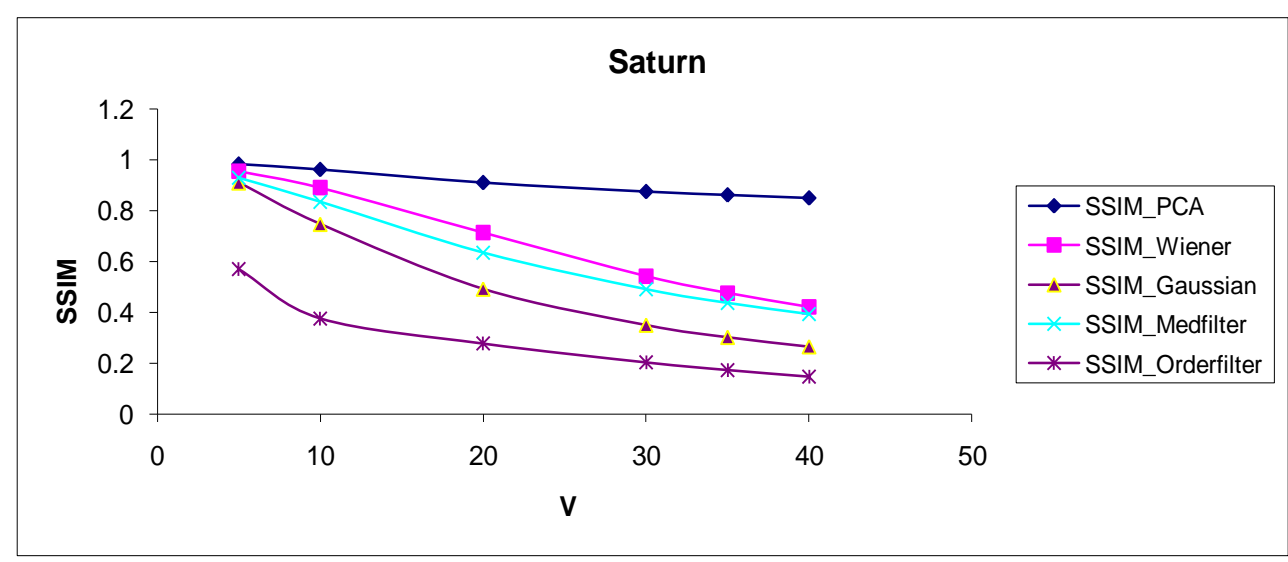

Fig.8: SSIM as a function of variance for Saturn.

It is clearly given that PCA gives a best PSNR and SSIM value among all.

Three test images used in the experiments are shown in Figs. 9-11, from the left first column: binary star, satellite and Saturn image. Second column, added Gaussian white noise to the original image with different variance levels $(v=5,10,20,30$, 35 and 40, respectively). Five denoising algorithms were used (third column: PCA, fourth column: Wiener filter, fifth column: Median filter, sixth column: Gaussian filter, seventh column: Order filter, respectively) for noise removal.

\section{Conclusions}

In order to provide unbiased results, evaluation with subjective measures requires careful selection of the test subject to correlated with human perception of image quality, the SSIM is one of the most commonly used measures for image visual quality assessment criteria.
As we know energy of noise evenly spreads over the whole data set, they can easily distinguish signal from noise over PCA domain. Experimental results shows LPGPCA method gives better performance, especially in image fine structure preservation, compared with other general denoising algorithms as shown as in Table 2. If the variance is high then second stage of LPG gives more PSNR and SSIM values. For lower variance images, first stage is sufficient to remove the noise as shown as in Table 1. To investigate a relationship between noise variance, PSNR and SSIM for each image Figs. 3-8 reveal this relation, Figs. 6-8 show a linear relationship between SSIM as a function of variance for PCA method. 

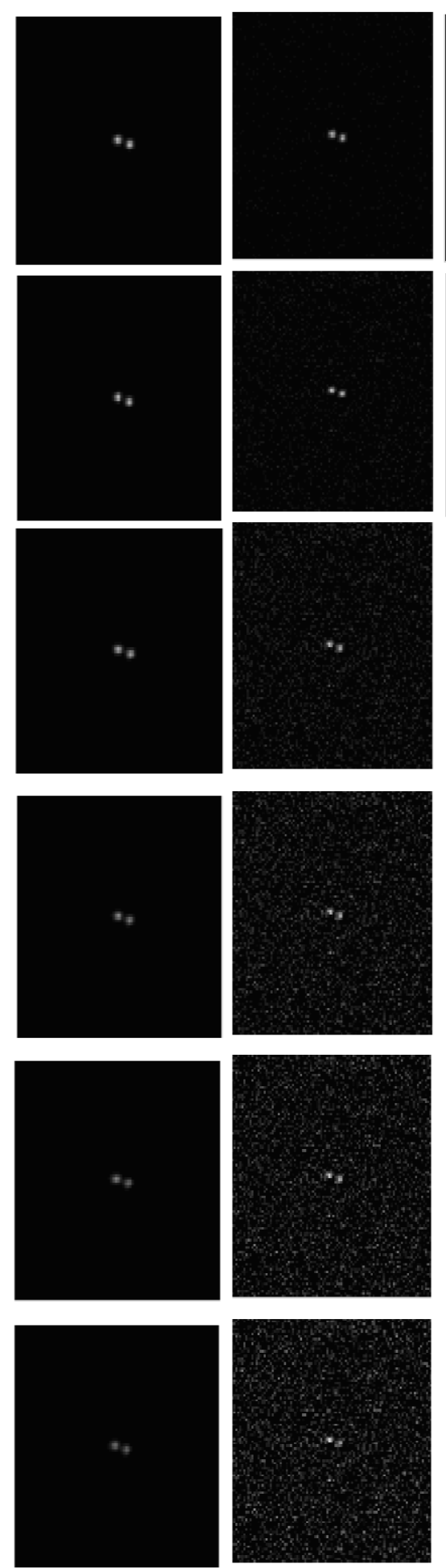
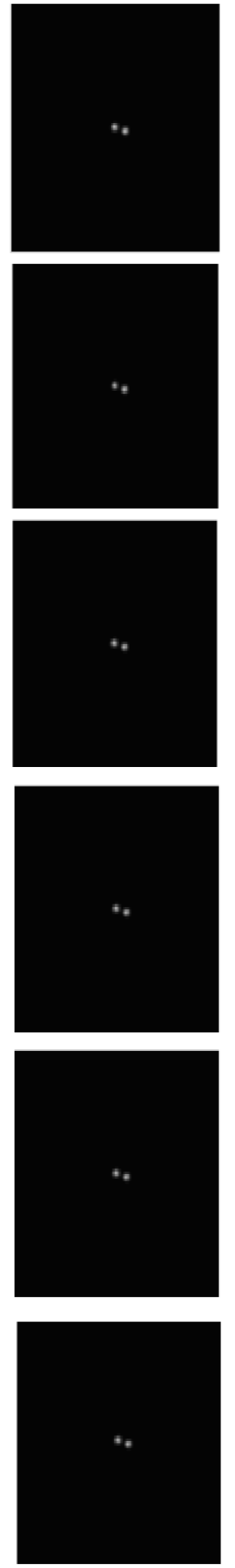

Fig.9: The denoising results of Binary star by different methods.
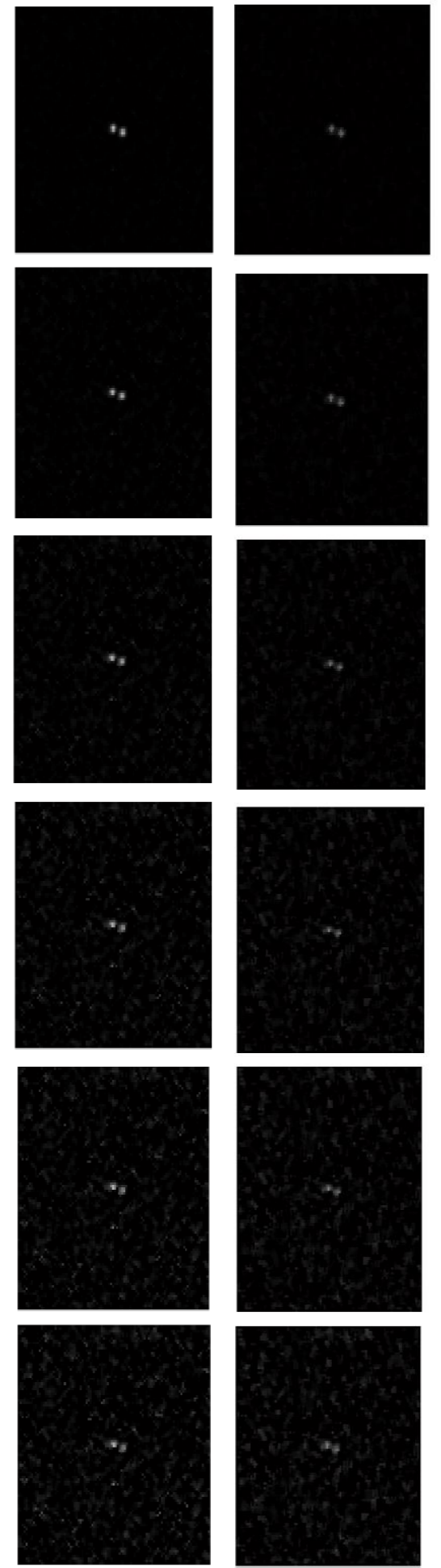
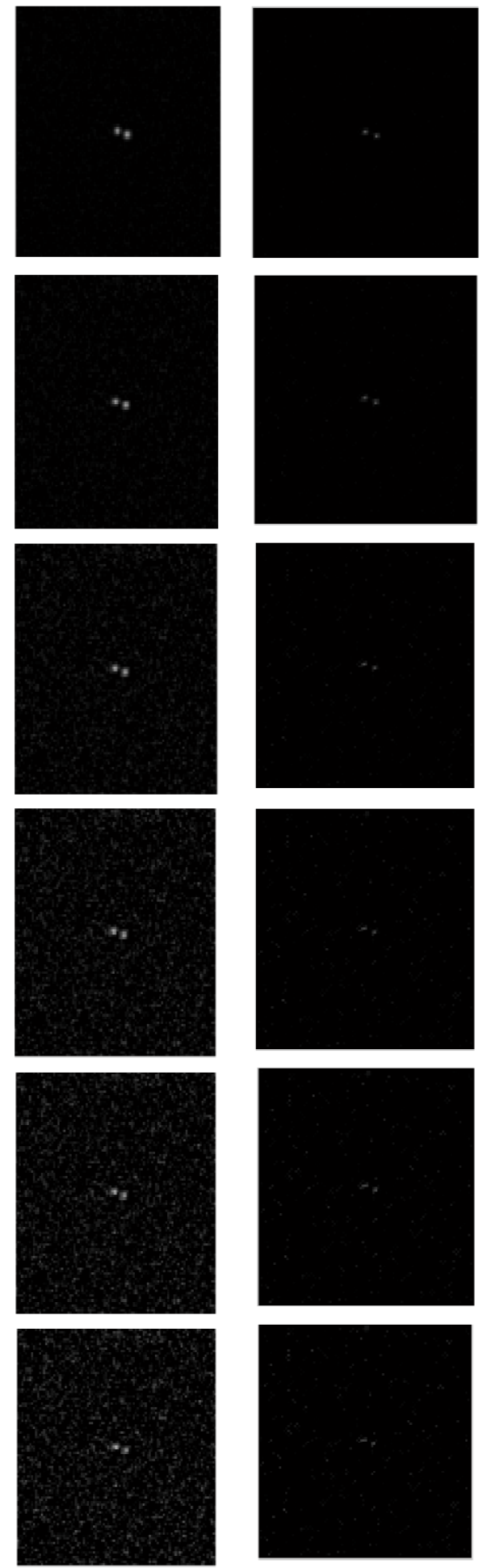
(a)

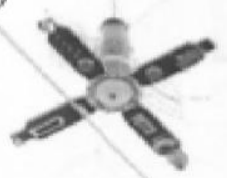

(a)

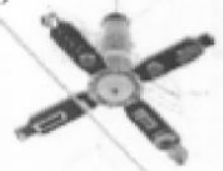

(a)

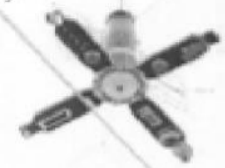

(a)

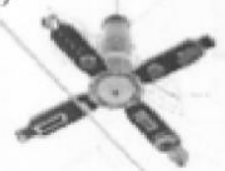

(a)

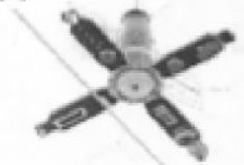

(a)

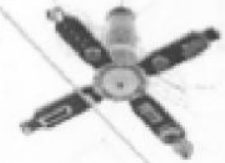

(a)

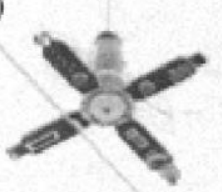

(a)

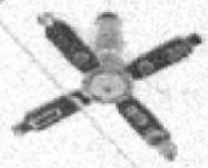

(a)

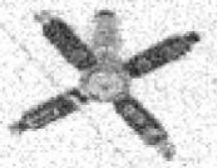

(a)

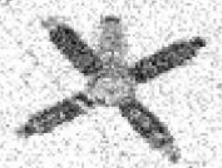

(a)
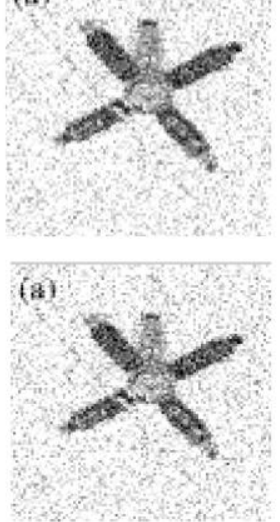

a)

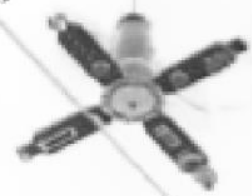

a)

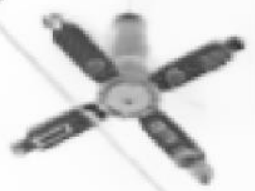

a)

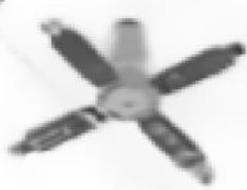

a)

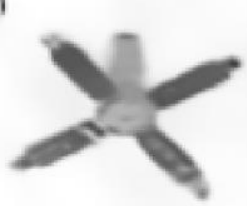

a)

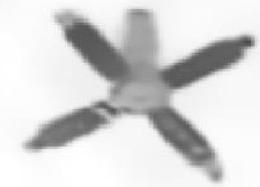

at

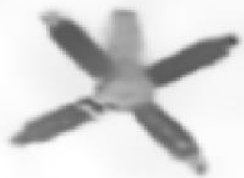

(a)

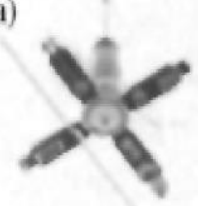

(a)

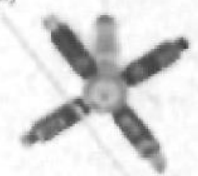

(a)

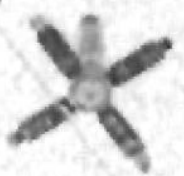

(a)

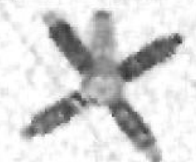

(a)

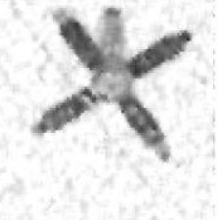

(a)

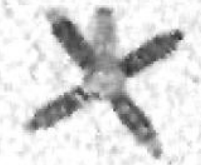

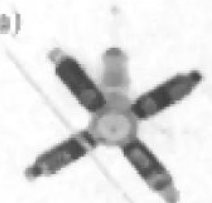

(a)

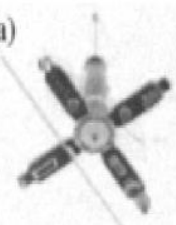

(a)

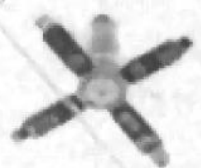

(a)

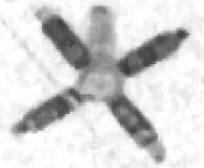

(a)

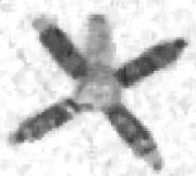

(it)
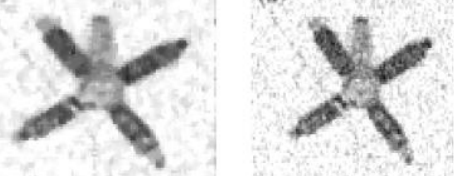

(i4)

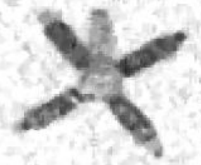

(a)

(a)

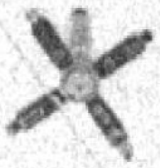

(a)

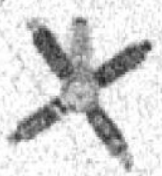

(a)

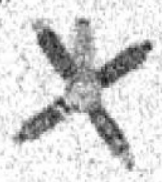

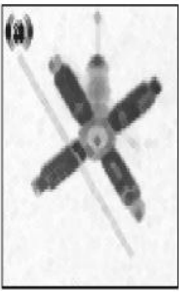

(a)
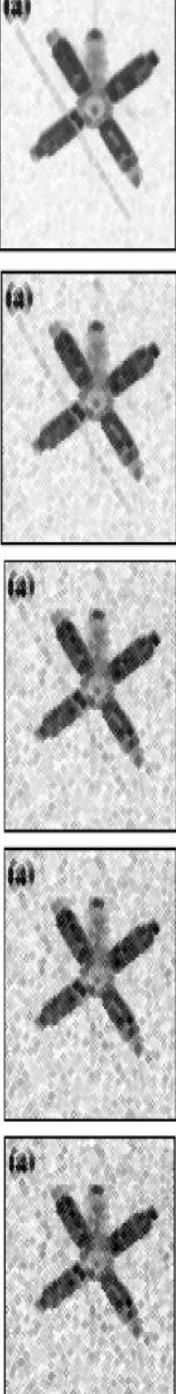

Fig.10: The denoising results of Satellite by different methods. 

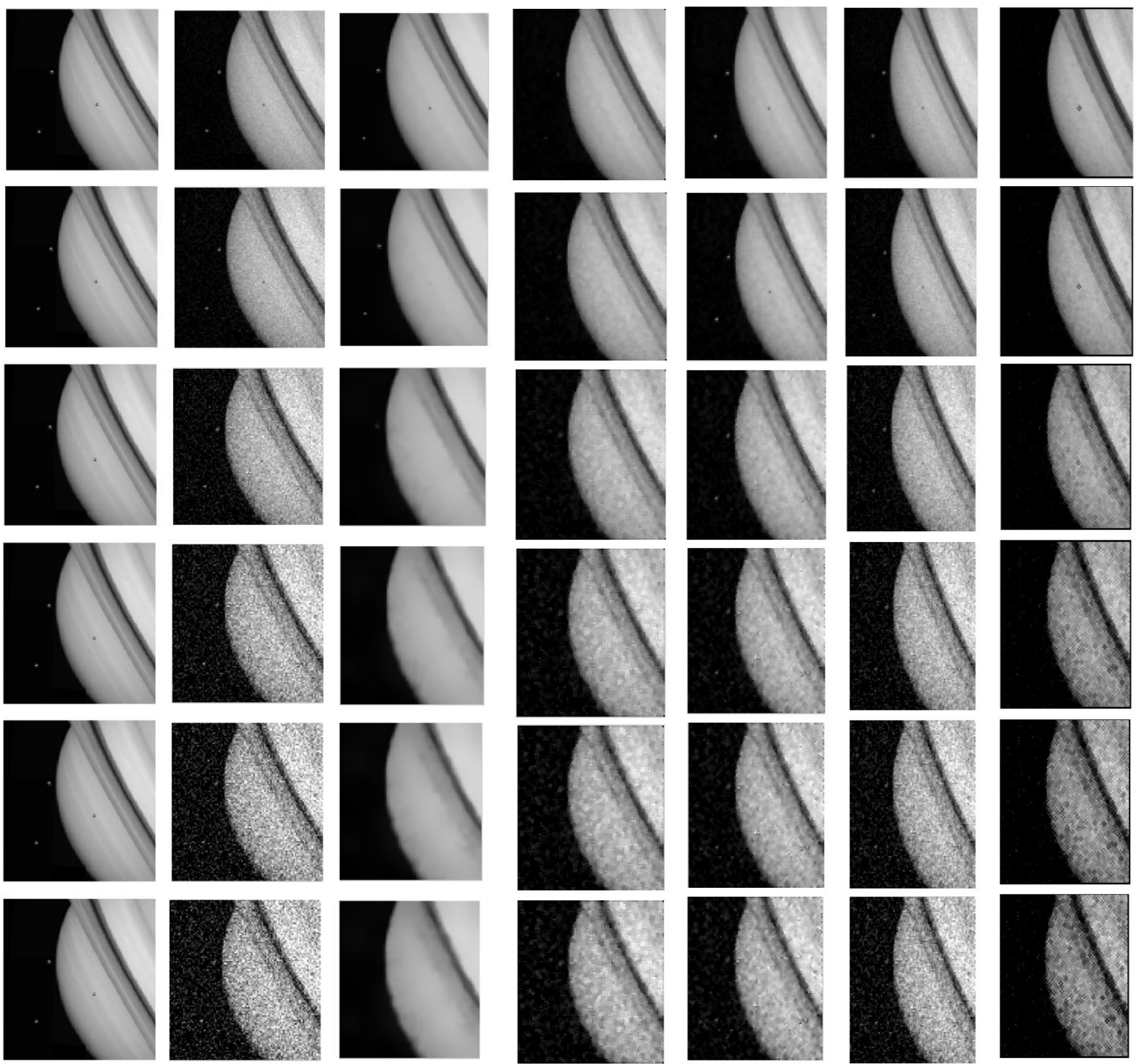

Fig.11: The denoising results of Saturn by different methods. 


\section{References}

[1] H. Zou, T. Hastie and R. Tibshirani, Journal of Computational and Graphical Statistics, 15, 2 (2006) 265-286.

[2] Y. M. Babu, M. V. Subramanyam, M. N. Prasad, Signal and Image Processing: An International Journal (SIPIJ), 3, 2, April (2012).

[3]A.A. Philip and M.M. Omotosho "Image processing Techniques for Denoising object Idintification and Feature Extraction" proceeding of the world congress on Engineering Vol. 3, WCE, London, U.K., (2013).

[4] M. Linting, J. J. Meulman, P. J. Groenen and A. J. van der Kooij, Psychological Methods, 12, 3 (2007) 359-379.

[5] L. E. Mujic, J. Vehı', M. Ruiz, M. Verleysen, W. Staszewski, K. Worden, Mechanical Systems and Signal Processing 22, (2008) 155-171.

[6] N. Fairley, "Principal Component Analysis", Casa Software Ltd., (2004).

[7] S. Stowell, "Instant R: An Introduction to R for Statistical Analysis", Published by Jotunheim Publishing, (2012).

[8] K. J. Peter, K. S. Kannan, S. Arumugan, G. Nagarajan, "Two-stage image denoising by Principal Component Analysis with Self Similarity pixel Strategy", IJCSNS
International Journal of Computer Science and Network Security, 11, 5, May (2011).

[9] S. Pal, R. Mahakud, M. Sahoo, "PCA based Image Denoising using LPG", IJCA Special Issue on "2nd National ConferenceComputing, Communication and Sensor Network" CCSN, (2011).

[10] S. E. Umbaugh, "Computer Vision and Image Processing", Prentice Hall PTR, (1998).

[11] I. T. Jolliffe, "Principal Component Analysis", Second edition Springer Series in Statistics. New York: Springer-Verlag New York, (2002).

[12] L. Zhang, W. Dong, D. Zhang, G. Shi, Elsevier-Pattern Recognition, 43, (2010) 1531-1549.

[13] H. Zhou, J. Wu and J. Zhang, "Digital Image Processing", part 1, Ventus Publishing ApS, (2012).

[14] M. S. Nixon and A. S. Aguado, "Feature Extraction and Image Processing", second edition, Academic Press is an imprint of Elsevier, (2008).

[15] Z. Wang, A. C. Bovik, H. R. Sheikh and E. P. Simoncelli, "Image Quality Assessment: From Error Measurement to Structural Similarity", IEEE Transactions on Image Processing, 13, 1, January (2004). 